\title{
Innovación docente para el prácticum II de podología general
}

\author{
ANa JuANa PÉREZ BELLOSO \\ Departamento de Podología \\ Universidad de Sevilla \\ aperez30@us.es \\ ORCID: https://orcid.org/0000-0003-3188-2010 \\ D.O.I.: http://dx.doi.org/10.12795/JDU.2018.i01.13 \\ Pp.: 230-242
}

\section{Resumen}

La aplicación del ciclo de mejora se ha realizado en una clase práctica de cinco horas de duración donde se han combinado sesiones clínicas y prácticas reales con pacientes. El mapa de contenidos se ha centrado en el acto terapéutico de la quiropodia y en completar de forma adecuada las historias clínicas. El modelo metodológico aplicado se ha basado fundamentalmente en el periodo práctico combinándose triangularmente con el trabajo de casa y los informes de casos. Como cuestionario inicial y final se ha utilizado un video que ha servido para comparar el comportamiento de los estudiantes el acto terapéutico inicial y final. La evaluación del aprendizaje de los estudiantes, comparando sus conocimientos iníciales y finales ha sido bastante llamativa, pues la mejora ha sido muy significativa. En un futuro intentaré aplicar la docencia tal y como he aprendido en este ciclo pues los resultados han sido muy positivos y gratificantes no solo para mí como docente sino también para los alumnos. 
Palabras claves: quiropodia, mapa de contenidos, cuestionarios, evaluación, ciclo de mejora.

\section{Descripción del contexto de la intervención}

La experiencia se ha realizado en una clase práctica de 5 horas de duración y 8 alumnos, en donde se han combinado sesiones clínicas y prácticas reales con pacientes que acuden al área clínica de podología. Las sesiones clínicas se realizan sobre pacientes que se hayan tratado en la práctica del día anterior, se elegirá un caso interesante, caso que tendrán que trabajar en casa buscando bibliografía sobre las patologías que tuviese el paciente, el tratamiento aplicado y si es posible su evolución.

Me da la sensación que lo que menos les gusta a los alumnos es preparar las sesiones clínicas, sobre todo por el hecho de trabajarlas en casa, sin embargo durante las prácticas con pacientes los he visto bastante interesados y atentos a las explicaciones y correcciones que les he ido detallando.

Esta sensación que tengo sobre las sesiones clínicas la interpreto por el grado de excitación y entusiasmo que se le ve al alumno, que es diferente al que aparece durante las prácticas.

Si es cierto que durante las prácticas con pacientes existen periodos en los que el alumno no tiene pacientes para atender y debe irse con otro compañero mientras espera su siguiente paciente. Durante ese tiempo también percibo un deterioro del interés por el aprendizaje.

Pienso que lo que más le cuesta de las sesiones clínicas es el tiempo que le tienen que dedicar a la búsqueda bibliográfica. Puesto que es queja por parte de la mayoría de los alumnos del poco tiempo que tienen entre las clases teóricas y prácticas. 


\section{Diseño previo del Ciclo de Mejora Docente}

Esta asignatura consta de 1 hora para sesiones clínicas y 4 para asistencia a pacientes reales. Es una asignatura que engloba muchos aspectos genéricos de la clínica Podológica, pero mi interés concreto es que una vez finalizado el curso los alumnos sepan rellenar de forma completa y ordenada una historia clínica y las fases secuenciales completas del tratamiento quiropodológico.

El sistema que vamos a aplicar para saber si el segundo ciclo de mejora fue o no efectivo es el siguiente:

- Para responder al objetivo "rellenar de forma completa y ordenada una historia clínica" se van a comparar la primera historia clínica que los alumnos rellenan con la última. De esta forma observaremos las diferencias y si ha habido mejora en dicho procedimiento.

- Para responder al objetivo "fases secuenciales completas del tratamiento quiropodológico", se gravará al alumno en su primera quiropodia y en la última. Al final del ciclo de mejora cada alumno visionará el primer y último vídeo de otro compañero e identificará y anotará las diferencias significativas.

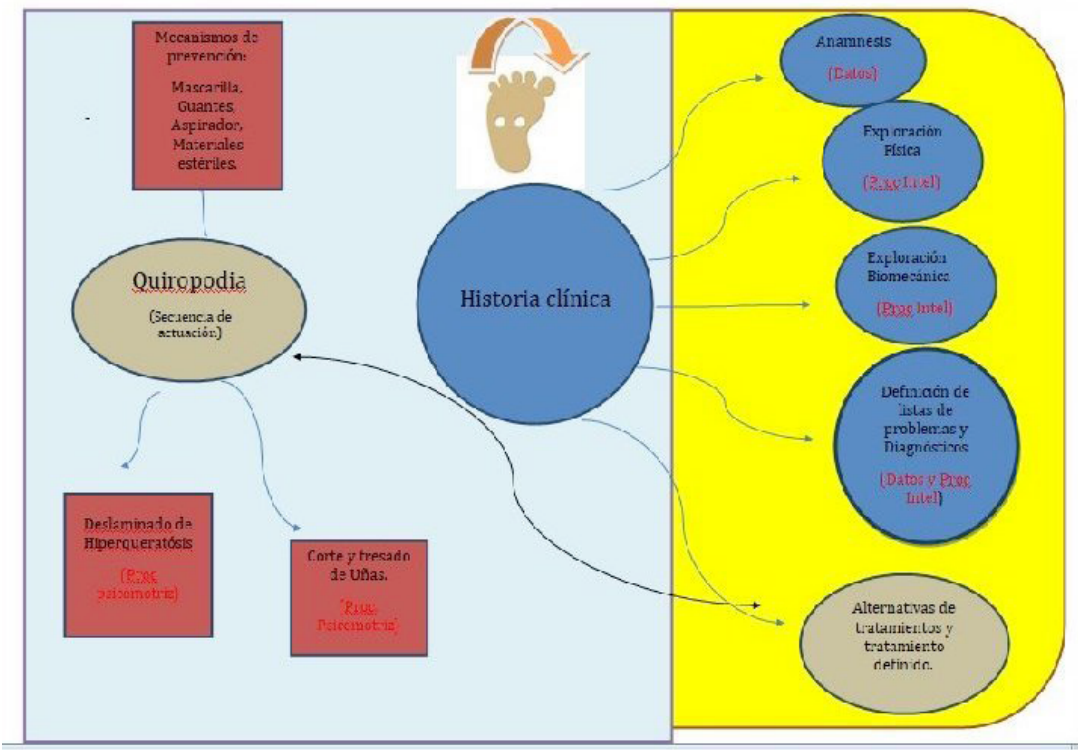

Figura 1.Mapa de contenidos del tema

Jornadas de Formación e Innovación Docente del Profesorado | № 1 (2018) Esta obra se distribuye con la licencia Creative Commons Reconocimiento-NoComercial-SinObraDerivada Internacional (CC BY-NC-ND 4.0.) 
El mapa de contenidos muestra esencialmente los contenidos intelectuales, procedimentales y de datos y la conexión existente entre ellos. El contenidofundamental es la historia clínica de ahí situarla en el centro del mapa, a partir de aquí salen las fases que se deben seguir para rellenar de forma completa y ordenada una historia clínica, dentro de estas fases encontramos la quiropodia, una fase del tratamiento importante en nuestra profesión.

Es importante que el tratamiento de la quiropodia sea completamente comprendido y que interioricen de forma sistemática lasfases secuenciales completas del tratamiento quiropodológico. Estas fases quedan representadas en el mapa de contenidos, aquellas que son fundamentales en este tratamiento y el orden secuencial del mismo, que queda representada con la imagen del pie.

Jornadas de Formación e Innovación Docente del Profesorado | № 1 (2018) Esta obra se distribuye con la licencia Creative Commons 
Modelo metodológico posible

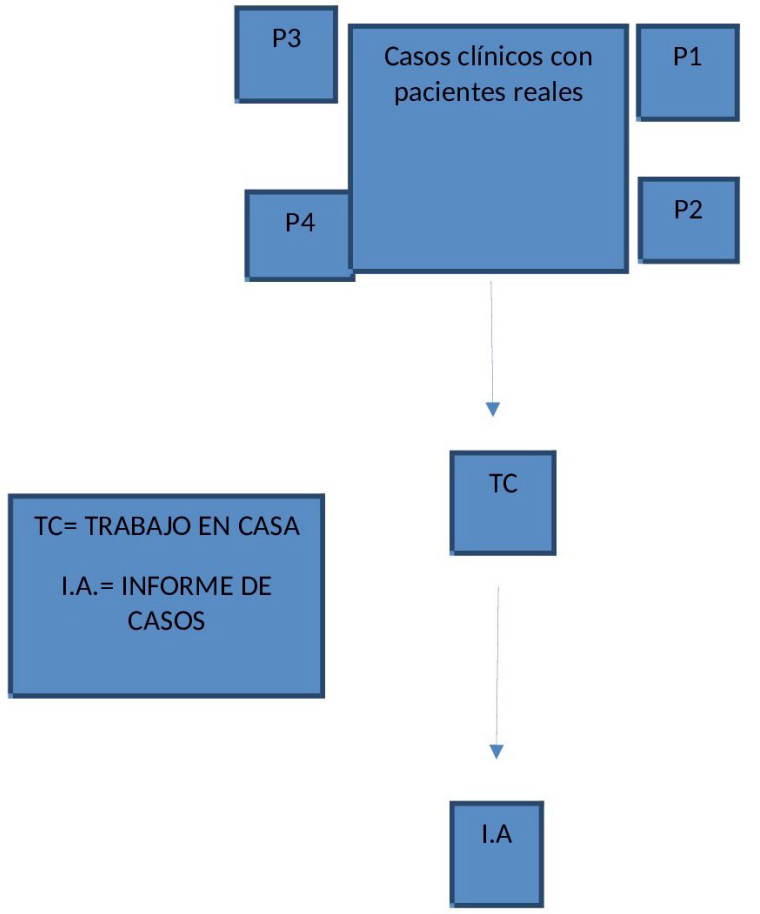

Figura 2. Modelo metodológico posible

Esta metodología está diseñada para intentar aprovechar al máximo la disponibilidad de pacientes reales durante las 5 horas de duración de la clase, de forma que el mayor aprendizaje se da en la práctica efectiva sobre pacientes y en la hora que se dedica al intercambio de conocimientos sobre patologías que presentaban los pacientes tratados.

La secuencia de actividades programada para este Ciclo de Mejora se presenta a continuación:

Jornadas de Formación e Innovación Docente del Profesorado | № 1 (2018) Esta obra se distribuye con la licencia Creative Commons 
Tabla 1: Secuencias de actividades del ciclo de mejora

\begin{tabular}{|l|l|c|}
\hline Actividad 1 & \multicolumn{1}{|c|}{ Trabajo en casa (TC) } & Duración \\
\hline $\begin{array}{l}\text { En esta actividad dos de los ocho alum- } \\
\text { nos (los dos alumnos que hayan tenido } \\
\text { los pacientes más interesantes) trabajan } \\
\text { en casa realizando búsquedas bibliográ- } \\
\text { ficas sobre el paciente que han traba- } \\
\text { jado durante las prácticas, de forma que } \\
\text { nos expongan: bibliografía sobre la pato- } \\
\text { logía que presentaban, presentación del } \\
\text { caso clínico con la descripción de su his- } \\
\text { toria clínica, tratamiento y evolución. }\end{array}$ & $\begin{array}{c}\text { Indetermi- } \\
\text { nado }\end{array}$ \\
\hline Recursos: PowerPoint, Internet, Libros de texto \\
\hline
\end{tabular}

\begin{tabular}{|l|l|l|}
\hline Actividad 2 & Recogida de firma de los alumnos & Duración5' \\
\hline Recursos: Lista de clase & \\
\hline
\end{tabular}

\begin{tabular}{|l|l|l|}
\hline Actividad 3 & \multicolumn{1}{|c|}{$\begin{array}{c}\text { Exposición del caso clínico } \\
\text { 10 Alumno (I.A.) }\end{array}$} & Duración \\
\hline & $\begin{array}{l}\text { Exposición del caso clínico preparado } \\
\text { durante el trabajo en casa }\end{array}$ & $15^{\prime}$ \\
\hline Recursos: Ordenador & \\
\hline
\end{tabular}

\begin{tabular}{|l|l|l|}
\hline Actividad 4 & $\begin{array}{l}\text { Debate del caso clínico expuesto. 10 } \\
\text { Alumno (I.A.) }\end{array}$ & Duración \\
\hline & $\begin{array}{l}\text { Todos los alumnos debaten sobre el } \\
\text { caso clínico expuesto, yo coordino y } \\
\text { animo a debatir sobre diversas alternati- } \\
\text { vas de tratamiento, complemento el caso } \\
\text { expuesto asociándolo a otras patologías } \\
\text { para que los alumnos propongan varias } \\
\text { alternativas de tratamiento cuando se } \\
\text { encuentran con dichas patologías }\end{array}$ & \\
\hline Recursos: & & 15' \\
\hline
\end{tabular}

Jornadas de Formación e Innovación Docente del Profesorado I № 1 (2018)

cC (i) E Esta obra se distribuye con la licencia Creative Commons Reconocimiento-NoComercial-SinObraDerivada $\quad 4.0$ Internacional (CC BY-NC-ND 4.0.) 


\begin{tabular}{|l|l|c|}
\hline Actividad 5 & \multicolumn{1}{|c|}{$\begin{array}{c}\text { Exposición del caso clínico } \\
\text { 20 Alumno (I.A.) }\end{array}$} & Duración \\
\hline & $\begin{array}{l}\text { Exposición del caso clínico preparado } \\
\text { durante el trabajo en casa. }\end{array}$ & $15^{\prime}$ \\
\hline Recursos: Ordenador & \\
\hline
\end{tabular}

\begin{tabular}{|l|c|c|}
\hline Actividad 6 & $\begin{array}{c}\text { Debate del caso clínico expuesto. 20 } \\
\text { Alumno (I.A.) }\end{array}$ & Duración \\
\hline & Igual que la actividad 4 & $15^{\prime}$ \\
\hline Recursos: & & \\
\hline
\end{tabular}

\begin{tabular}{|c|c|c|}
\hline Actividad 7 & Recepción de pacientes (Casos Clínicos) & Duración \\
\hline & $\begin{array}{l}\text { Las auxiliares me dan el listado de } 4 \text { pa- } \\
\text { cientes que vienen a las } 9 \text { horas y se re- } \\
\text { parten a } 4 \text { de los alumnos, los otros } 4 \\
\text { van de apoyo con los que tienen pacien- } \\
\text { tes y van rotando para poder ver a todos } \\
\text { los pacientes. }\end{array}$ & $10^{\prime}$ \\
\hline \multicolumn{3}{|l|}{ Recursos: } \\
\hline \multirow[t]{2}{*}{ Actividad 8} & $\begin{array}{l}\text { Atención del alumno al paciente } \\
\text { (10grupo de } 4 \text { alumnos) }\end{array}$ & Duración \\
\hline & $\begin{array}{l}\text { Durante esta actividad el alumno } \\
\text { atiende al paciente, le realiza una histo- } \\
\text { ria clínica completa con exploración arti- } \\
\text { cular y biomecánica y posteriormente se } \\
\text { enfoca en la patología que presenta, ad- } \\
\text { ministrando el tratamiento oportuno. } \\
\text { Yo voy pasando por cada gabinete para } \\
\text { ir resolviendo dudas y controlar la ac- } \\
\text { tuación del alumno. }\end{array}$ & 1'5 horas \\
\hline \multicolumn{3}{|l|}{ Recursos: } \\
\hline Actividad 9 & Recepción de pacientes (Casos Clínicos) & Duración \\
\hline
\end{tabular}

Jornadas de Formación e Innovación Docente del Profesorado I № 1 (2018) (c) (i) $\odot$ Esta obra se distribuye con la licencia Creative Commons Reconocimiento-NoComercial-SinObraDerivada $\quad 4.0$ Internacional (CC BY-NC-ND 4.0.) 
Se reparten los siguientes 4 pacientes a los 4 alumnos restantes

Recursos:

\begin{tabular}{|l|l|c|}
\hline Actividad 10 & \multicolumn{1}{|c|}{$\begin{array}{c}\text { Atención del alumno al paciente } \\
\text { (2ogrupo de 4 alumnos) }\end{array}$} & Duración \\
\hline Igual que la actividad 8 & 1.5 horas \\
\hline Recursos: & \multicolumn{1}{|c|}{ Conclusiones de las prácticas } & Duración \\
\hline Actividad 11 & $\begin{array}{l}\text { Se resuelve y debate sobre dudas apare- } \\
\text { cidas durante la atención al paciente. }\end{array}$ & 20' \\
\hline & $\begin{array}{l}\text { Reparto de sesiones clínicas para la si- } \\
\text { guiente sesión } \\
\text { Recursos: }\end{array}$ & $\begin{array}{l}\text { Duración } \\
\text { los más interesantes para prepararlos } \\
\text { para la sesión clínica siguiente }\end{array}$ \\
\hline Actividad 12 & \begin{tabular}{l} 
10' \\
\hline Recursos:
\end{tabular} & \\
\hline
\end{tabular}

\section{Aplicación del Ciclo de Mejora Docente}

Comenzamos la clase con las sesiones clínicas tal y como indico en las actividades, una vez finalizadas y antes de comenzar las prácticas con los pacientes reales, expliqué a los alumnos que conforme se quedaran libres de los pacientes asignados, un becario o asistente honorario, se prestaría voluntariamente para que cada alumno realizase una quiropodia, mientras serian gravados con el móvil por dichos voluntarios.

Jornadas de Formación e Innovación Docente del Profesorado I № 1(2018) Reconocimiento-NoComercial-SinObraDerivada Internacional (CC BY-NC-ND 4.0.) 
También les explique, que para la siguiente clase (que dura otras 5 horas), en lugar de sesiones clínicas, realizaría yo personalmente una quiropodia para que ellos observaran el orden secuencial de la misma y a posteriori, al final de esta segunda clase, volverían a ser gravados realizando el mismo acto.

Las dos clases (ambas de 5 horas cada una y compuesta de 8 alumnos), trascurrieron según lo previsto. Las prácticas con los pacientes reales fueron bastante bien, en ellas los becarios y yo pasábamos por los distintos gabinetes revisando y recordando el orden secuencial.

Con respecto al correcto desarrollo de la historia clínica, he cogido la primera historia rellenada por cada uno de los alumnos en la primera sesión y la última de la segunda para poder hacer una comparación de las mismas.

Por último les solicité que cada uno analizara sus videos, el primero y el último, y me elaboraran un informe con las diferencias observadas y los errores cometidos.

- Descripción analítica y conclusiones

Si analizamos bien el trascurso de las dos sesiones, los alumnos creo que se sintieron expectantes ante la novedad de gravarse en video.

Si es cierto que al gravarse con sus propios móviles y tenérmelos que enviar a mí, he tardado en poder recopilar todos los videos pero preferí realizarlo así por el tema de la protección de datos.

Ha sido una buena experiencia pues los alumnos al sentirse observados a través del video pusieron mucho más interés en realizarlo lo mejor posible, incluso una vez gravado me mandaban correos con actuaciones que se les había pasado hacer en el video y que se habían acordado después.

Por todo ello note mucha diferencia entre el primer y segundo video, con muchos cambios positivos en los 8 alumnos.

- Evaluación del aprendizaje de los estudiantes. 
Antes de comenzar con el análisis comentar que hemos dividido el video en dos cuestiones que son de interés sobre el aprendizaje del acto de la quiropodia:

1. Aplicación correcta del orden secuencial de la quiropodia

2. Aplicación correcta de las medidas de prevención.

Con respecto a rellenar de forma adecuada o no las historias clínicas hemos valorado:

1. Si la historia clínica está completamente elaborada.

2. Si el contenido se corresponde a cada punto de la historia clínica, es decir, sin el contenido es adecuado y acorde a las circunstancias del paciente.

Una vez recogidos los videos, los informes elaborados por los alumnos y las historias clínicas, el análisis inicial ha quedado de la siguiente manera:(1)

Aplicación correcta del orden secuencial de la quiropodia y medidas de prevención

Test inicial

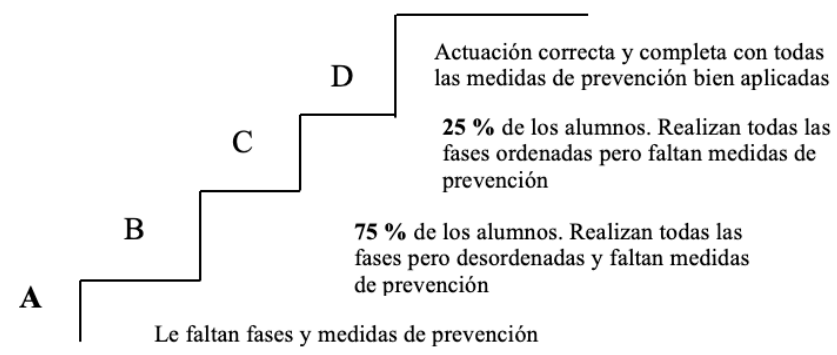

Test inicial

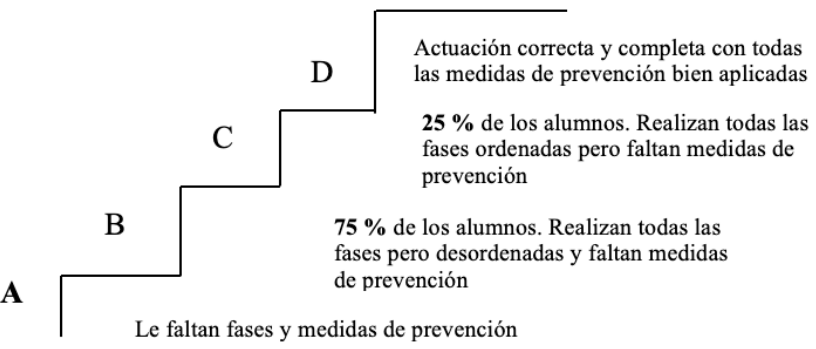

Jornadas de Formación e Innovación Docente del Profesorado | № 1 (2018)

Esta obra se distribuye con la licencia Creative Commons 
Test inicial

D Historia clínica completamente elaborada y con contenido adecuado

A

B C Historia clínica completamente elaborada

Test final

D $\quad \mathbf{2 5} \%$ Historia clínica completamente

12.5\%.Ni está completa ni acorde al paciente

Tabla 2. Progresión de cada alumno desde el cuestionario inicial al cuestionario final

\section{NIVELES DE DESARROLLO INICIALES Y FINALES}

\begin{tabular}{|l|c|c|c|c|c|c|}
\hline \multicolumn{2}{|c|}{} & \multicolumn{3}{|c|}{ PREGUNTA 1 } & \multicolumn{2}{c|}{ PREGUNTA 2 } \\
\hline sujeto 1 & $\mathrm{B}$ & $\mathrm{B}$ & $\Leftrightarrow$ & $\mathrm{B}$ & $\mathrm{C}$ & $\uparrow$ \\
\hline sujeto 2 & $\mathrm{B}$ & $\mathrm{B}$ & $\Leftrightarrow$ & $\mathrm{B}$ & $\mathrm{B}$ & $\Leftrightarrow$ \\
\hline sujet03 & $\mathrm{B}$ & $\mathrm{C}$ & $\uparrow$ & $\mathrm{B}$ & $\mathrm{C}$ & $\uparrow$ \\
\hline sujet04 & $\mathrm{B}$ & $\mathrm{C}$ & $\uparrow$ & $\mathrm{B}$ & $\mathrm{D}$ & $\uparrow$ \\
\hline sujet05 & $\mathrm{B}$ & $\mathrm{D}$ & $\uparrow$ & $\mathrm{A}$ & $\mathrm{B}$ & $\uparrow$ \\
\hline sujet06 & $\mathrm{B}$ & $\mathrm{C}$ & $\uparrow$ & $\mathrm{B}$ & $\mathrm{D}$ & $\uparrow$ \\
\hline sujeto 7 & $\mathrm{C}$ & $\mathrm{C}$ & $\Leftrightarrow$ & $\mathrm{B}$ & $\mathrm{B}$ & $\Leftrightarrow$ \\
\hline sujet08 & $\mathrm{C}$ & $\mathrm{D}$ & $\uparrow$ & $\mathrm{B}$ & $\mathrm{B}$ & $\Leftrightarrow$ \\
\hline
\end{tabular}

Si analizamos los resultados se observa claramente que la mayoría de los alumnos han evolucionado positivamente.En la tercera columna de cada apartado se muestra gráficamente (con una flecha) la evolución de cada sujeto, 
algunos de ellos no han evolucionado en la escalera pero la mayoría han ascendido de escalón a niveles de conocimientos altos dentro de la escalera de evolución

\section{Evaluación del Ciclo de Mejora Docente puesto en práctica}

Los modelos mentales de los alumnos varían ampliamente y resulta complicado conocerlos todos, si es cierto que las pruebasiníciales nos ayudan mucho a conocer estos modelos y a saber cómo poder enfocar una docencia centrada en los alumnos en sus inquietudes y dudas.

Para ello es importantísima la interacción con el alumno, dejarle claro desde primera hora como se desarrollaran las clases y las cuestiones más importantes que se van a tratar.

Está claro que clasificar los contenidos que se van han impartir es lo más importante, si algo me ha quedado claro en este seminario, es que más vale poco y bien, que mucho y mal. Para ello habrá que clasificar exhaustivamente los contenidos y jerarquizarlos. Posteriormente elaborar los mapas y laspruebasiníciales para poder definir el nivel de los alumnos y saber por dónde comenzar las clases y en que parte de la materia incidir.

Según entiendo los mejores modelos metodológicos son los que se centran en los alumnos y en sus hipótesis, y en donde ellos trabajan y resuelven casos reales.

Debido a que la mayor parte de mi docencia son prácticas clínicas con pacientes reales, me resulta mucho más sencillo aplicar este tipo de modelo ideal. Aun así, nunca había elaborado un modelo metodológico, ni elaborado preguntas, ni secuencia de actividades.

Esto me ha servido para cuadrar mucho mejor las prácticas, enfocar mucho más la docencia en lo que me interesa que los alumnos aprendan y que no se me olviden cuestiones que quería plantear a los alumnos que si no fuese así, en muchos casos se me olvidaban. 
Respecto a la evaluación, he observado una buena diferencia entre el diagnostico inicial y final de las ideas o modelos mentales de los estudiantes, una diferencia positiva que supongo que indica que el método docente aplicado ha funcionado razonablemente bien.

Las dificultades de aprendizaje, las escaleras de aprendizaje, los relatos sobre la práctica, el diario del profesor, los portafolios, la evaluación del diseño didáctico y del docente, etc., todos estos puntos nos permiten realizar una evaluación continuada, no solo del alumno, sino también del docente. Esta evaluación continuada nos permite ir realizando cambios en la docencia durante el transcurso de las clases a medida que vamos viendo la evolución de los alumnos. (Porlan, 2017)

Si es cierto que los alumnos no están acostumbrados a este tipo de docencia y la incertidumbre de cómo será la evaluación final siempre les ronda por la cabeza. Por ello, creo que sería necesario dejar claro cómo se va a realizar la evaluación final, que en mi caso, no se realizará con un examen, si no que se evaluarán las actitudes, participación y realización de tareas.

\section{Referencias bibliográficas}

1) Porlán, R.(Coord) (2017). Enseñanza universitaria. Cómo mejorarla. Madrid: Morata.

Jornadas de Formación e Innovación Docente del Profesorado | № 1 (2018) Esta obra se distribuye con la licencia Creative Commons 